\title{
Jiao-tai-wan for insomnia symptoms caused by the disharmony of the heart and kidney: a study protocol for a randomized, double- blind, placebo-controlled trial
}

Zeng Congcong ${ }^{1 \dagger}$, Liu Xi ${ }^{2+}$, Hu Lufeng ${ }^{3}$, Feng Yuan ${ }^{4}$, Xia Nengzhi ${ }^{5}$, Zeng Haihuan ${ }^{6}$, Luo Lin ${ }^{7}$, Ye Ren ${ }^{1 *}$ and Yuan Zhengzhong ${ }^{1 *}$

\begin{abstract}
Background: Insomnia seriously affects people's normal lives and work. However, effective treatment strategies are scarce. The purpose of this study is to explore the efficacy and safety of Jiao-tai-wan (JTW) for ameliorating insomnia symptoms caused by disharmony of the heart and kidney.

Design: This is a randomized, double-blind, placebo-controlled pilot clinical trial. A total of 124 participants suffering from insomnia symptoms will be randomly assigned to the JTW or placebo group in an equal ratio. The participants will be asked to take JTW or placebo granules twice a day for 1 week. All data will be gathered at baseline and at the end of the drug intervention. The primary outcome measures will be the mean change in the Pittsburgh Sleep Quality Index (PSQI) from baseline to the end of the drug intervention. Secondary outcome measures will include the altered sleep parameters in polysomnography, ${ }^{1} \mathrm{H}$-magnetic resonance spectroscopy $\left({ }^{1} \mathrm{H}\right.$ MRS) evaluation, the Disharmony of Heart and Kidney Scoring System score, and blood tests, including the levels of serum adenosine and melatonin. A laboratory test will be taken before and after treatment to assess the safety of JTW.
\end{abstract}

Discussion: The outcomes of this study will confirm the efficacy of JTW for the treatment of insomnia symptoms and will also be used to monitor the safety of JTW.

Trial registration: Chinese Clinical Trial Registry, ChiCTR1800019239. Registered on 1st November 2018.

Keywords: Jiao-tai-wan, Insomnia, Traditional herbal medicine, Randomized controlled trial, Study protocol, Pattern identification

\footnotetext{
* Correspondence: yeren601@163.com; wzyzz2008@126.com

${ }^{+}$Congcong Zeng and Xi Liu contributed equally to this work

'Department of Traditional Chinese Medicine, The First Affiliated Hospital of

Wenzhou Medical University, Nanbaixiang, Ouhai District, Wenzhou 325035,

Zhejiang, China
}

Full list of author information is available at the end of the article

(c) The Author(s). 2020 Open Access This article is licensed under a Creative Commons Attribution 4.0 International License, which permits use, sharing, adaptation, distribution and reproduction in any medium or format, as long as you give appropriate credit to the original author(s) and the source, provide a link to the Creative Commons licence, and indicate if changes were made. The images or other third party material in this article are included in the article's Creative Commons licence, unless indicated otherwise in a credit line to the material. If material is not included in the article's Creative Commons licence and your intended use is not permitted by statutory regulation or exceeds the permitted use, you will need to obtain permission directly from the copyright holder. To view a copy of this licence, visit http://creativecommons.org/licenses/by/4.0/. The Creative Commons Public Domain Dedication waiver (http://creativecommons.org/publicdomain/zero/1.0/) applies to the data made available in this article, unless otherwise stated in a credit line to the data. 


\section{Background}

Sleep is essential to human health, but unfortunately, nearly one-third to one-quarter of the population in resource-poor countries suffer from insomnia at some level [1, 2]. With the increasing incidence of insomnia, there are different degrees of depression, anxiety, and other psychological diseases, which seriously affect the quality of a person's daily life [3]. Psychological and behavioral therapy, Western medicinal therapy, and traditional medicinal therapy are the main treatments for insomnia [4]. Many clinical studies have supported the first two treatments; however, some risks of adverse reactions and tolerance have been reported. In addition, dependence and addiction seriously impact clinical efficacy. For instance, the long-term use of zolpidem, which is relatively safe and frequently prescribed for the treatment of transient insomnia, will increase the risk of dementia [5]. Thus, interest in using traditional medicinal therapies is increasing, especially in traditional herbal medicine [6].

The superiority and soul of traditional East Asian medicine is the system called "pattern identification," which is used to diagnose and treat diseases based on the symptoms and the signs observed in patients. According to this system, insomnia has many different patterns, but disharmony of the heart and kidney is the dominant pattern [7, 8]. Moreover, Jiao-tai-wan (JTW) has been commonly used for the management of insomnia with a disharmony of heart and kidney pattern for centuries in China, Korea, and Japan [9].

In a review article by Yeung et al. [10], JTW was listed as one of the 10 most frequently examined standardized traditional herbal formulas for insomnia. A clinical study conducted in China showed that administration of JTW for 60 consecutive days could improve the Pittsburgh Sleep Quality Index (PSQI) [11]. Lu et al. [12] reported a significant decrease in scores in the Disharmony of Heart and Kidney Scoring System. Another study [13] demonstrated the effects of JTW in detail by alleviating the symptoms of palpitation and dizziness caused by the disharmony of the heart and kidney.

Although recent clinical studies have shown the efficacy of JTW in the treatment of insomnia, a randomized, double-blind, placebo-controlled pilot clinical trial to confirm its efficacy has not yet been undertaken. We set up this study to better clarify the efficacy, safety, and feasibility of JTW for treating insomnia caused by disharmony of the heart and kidney and to provide data for evidence-based clinical practice.

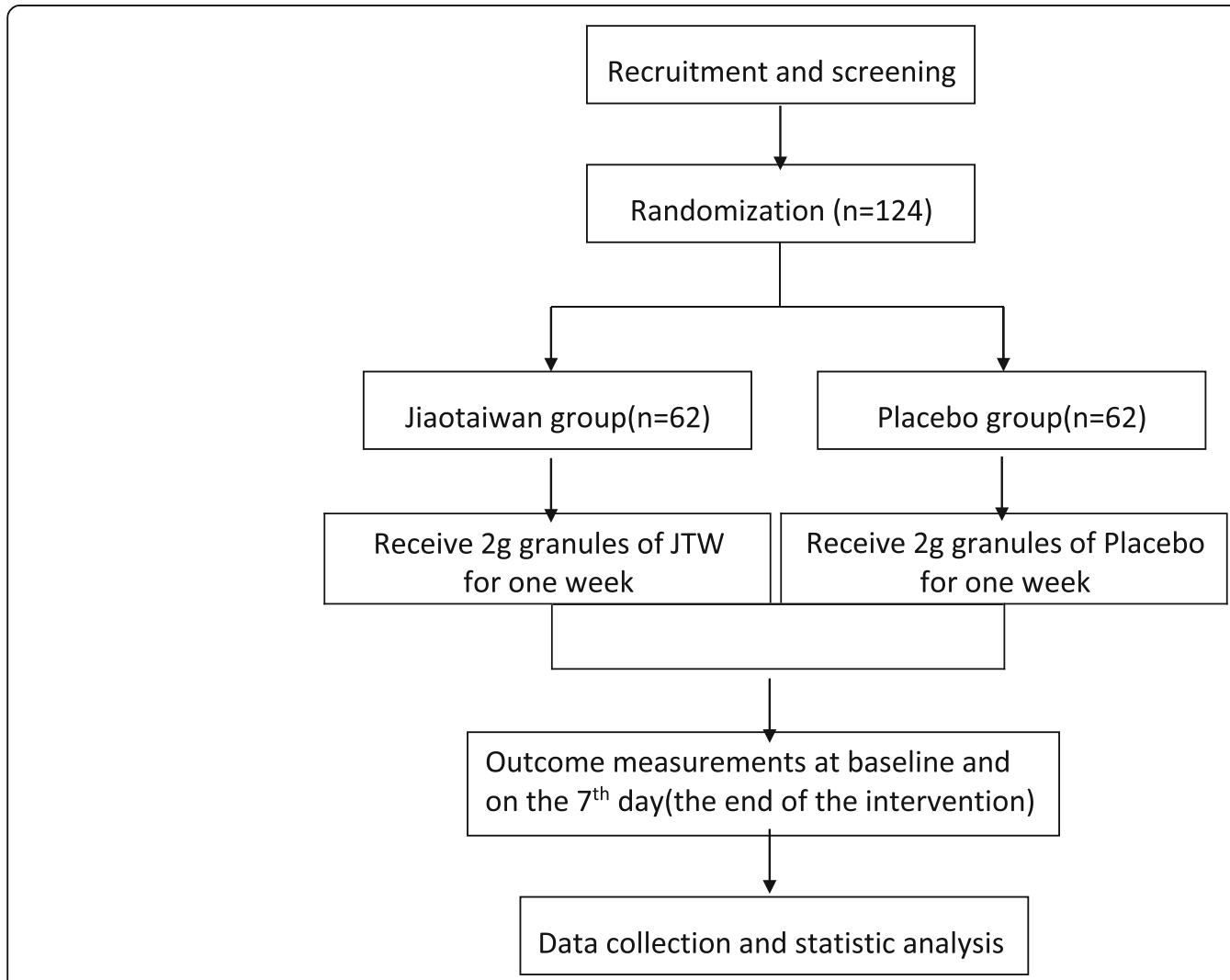

Fig. 1 Flow chart of the study design 


\begin{tabular}{|c|c|c|c|c|c|c|c|c|c|c|c|}
\hline \multirow[b]{2}{*}{ Time point } & \multicolumn{2}{|c|}{ Baseline } & \multicolumn{7}{|c|}{ 7-day Intervention } & \multicolumn{2}{|c|}{ Assess } \\
\hline & $\begin{array}{l}\text { DAY } \\
-2\end{array}$ & $\begin{array}{l}\text { Day } \\
-1\end{array}$ & $\begin{array}{l}\text { Day } \\
1\end{array}$ & $\begin{array}{l}\text { Day } \\
2\end{array}$ & $\begin{array}{l}\text { Day } \\
3\end{array}$ & $\begin{array}{l}\text { Day } \\
4\end{array}$ & $\begin{array}{l}\text { Day } \\
5\end{array}$ & $\begin{array}{l}\text { Day } \\
6\end{array}$ & $\begin{array}{l}\text { Day } \\
7\end{array}$ & $\begin{array}{l}\text { Day } \\
1\end{array}$ & \begin{tabular}{|l} 
Day \\
2
\end{tabular} \\
\hline \multicolumn{12}{|l|}{ Enrollment } \\
\hline Eligibility screen & $x$ & & & & & & & & & & \\
\hline Informed consent & $x$ & & & & & & & & & & \\
\hline Allocation & & $x$ & & & & & & & & & \\
\hline \multicolumn{12}{|l|}{ Intervention } \\
\hline Treatment group & & & $x$ & $x$ & $x$ & $x$ & $x$ & $x$ & $x$ & & \\
\hline Placebo group & & & $x$ & $x$ & $x$ & $x$ & $x$ & $x$ & $x$ & & \\
\hline \multicolumn{12}{|l|}{ Assessments } \\
\hline Socio-demographics & $x$ & & & & & & & & & & \\
\hline Medical history & $x$ & & & & & & & & & & \\
\hline Physical examination & $x$ & & & & & & & & & & $x$ \\
\hline $\begin{array}{l}\text { Concomitant } \\
\text { treatment }\end{array}$ & & & $x$ & $x$ & $x$ & $x$ & $x$ & $x$ & $x$ & & \\
\hline $\mathrm{PSQI}^{\mathrm{b}}$ & $x$ & & & & & & & & & $x$ & \\
\hline Polysomnography & $x$ & $x$ & & & & & & & & $x$ & $x$ \\
\hline${ }^{1} \mathrm{H}-\mathrm{MRS}^{\mathrm{c}}$ & $x$ & & & & & & & & & $x$ & \\
\hline DHKSS $^{\text {d }}$ & $x$ & & & & & & & & & $x$ & \\
\hline Blood tests ${ }^{e}$ & & $x$ & & & & & & & & & $x$ \\
\hline Medication adherence & & & $x$ & $x$ & $x$ & $x$ & $x$ & $x$ & $x$ & & \\
\hline Adverse events & & & $x$ & $x$ & $x$ & $x$ & $x$ & $x$ & $x$ & $x$ & $x$ \\
\hline Blinding test & & & & & & & & & & & $x$ \\
\hline
\end{tabular}

Abbreviations. PSQI:Pittsburgh Sleep Quality Index; ${ }^{1} \mathrm{H}-\mathrm{MRS}$ : $1 \mathrm{H}^{-}$-magnetic resonance spectroscopy;DHKSS:Disharmony of Heart and Kidney Scoring System.

Physical examination ${ }^{\mathrm{a}}$ : breath rate, heart rate, temperature, blood pressure, weight, routine urine, routine blood test, liver, kidney function tests, and electrocardiogram.

Blood tests: The levels of adenosine (AD) and melatonin (N-acety1-5methoxytryptamine).

Fig. 2 SPIRIT schedule for enrollment, treatment, and assessments

\section{Methods and design \\ Study design}

This is a parallel group randomized superiority clinical trial comparing JTW to placebo for efficacy in treating insomnia. The choice of using a placebo for the trial in order to test the rationality of the study design and to explore the effectiveness of JTW. Eligible participants will be randomly assigned to either the JTW or placebo group in an equal proportion. The drug intervention will last for 1 week. Figure 1 briefly shows the study flow chart and Fig. 2 enumerates the treatment schedule and outcome measures. The study adheres to the Standard Protocol Items: Recommendations for Interventional Trials (SPIRIT 2013) checklist [14] (Additional file 1). 


\section{Participants}

\section{Study population}

Patients suffering from insomnia symptoms caused by the disharmony of the heart and kidney will be recruited from the Traditional Chinese Medicine Department and Sleep Centre of the First Affiliated Hospital of Wenzhou Medical University in Wenzhou, Zhejiang Province, China. To recruit target participants, advertisements will be distributed using flyers, Wechat, and online and offline bulletin boards of the hospital.

\section{Inclusion criteria}

1. Participants are aged $18-60$ years, either male or female, and with a level of education above junior high school.

2. Insomnia is almost the only symptom, including difficulty sleeping, deep sleep, dreaminess, earlymorning awakening, trouble going back to sleep, feeling tired after waking up, or daytime sleepiness (excluding secondary insomnia).

3. A PSQI score of 7 or higher according to the Chinese Classification and Diagnostic Criteria of Mental Disorders-3 Version, CCMD-3 [15].

4. A Disharmony of Heart and Kidney Scoring System score of 9 or higher.

5. Signed informed consent before treatment.

\section{Exclusion criteria}

1. Insomnia caused by physical diseases or lifestyle changes or environmental disturbances.

2. Affective disorders, anxiety disorder, depression, schizophrenia, and any other serious mental disorders.

3. Suffering from somatic diseases that affect the central nervous system

4. Serious heart, liver, kidney, and hematopoietic system diseases, abnormal liver and kidney function.

5. Regular use of antipsychotic drugs in the last month.

6. Use of medicine to treatment sleeping disorders in the past week.

7. Allergic to a certain ingredient of the drug involved in this trial or suffering from allergies.

8. Alcohol abuse and/or psychotropic drug addiction.

9. Pregnancy or lactation.

\section{Randomization and allocation concealment}

We will apply block randomization to generate a sequence of labels (A or $\mathrm{B}$ ) at 1:1 ratio, numbered from 001 to 124 . This randomization approach forces balance between treatment and placebo groups over time as the recruitment proceeds. The pharmaceutical company will prepare the trial intervention packages according to the labeled sequence (A or B), numbered from 001 to 124 , all in uniform plastic packages. The study coordinator will be blinded to the group assignment and will give participants the numbered package of intervention according to their visiting time sequence at baseline visit (e.g. the first participant will get the package numbered 001, the second participant will get packaged 002, and so on). Thus, the allocation concealment will be maintained throughout the study. The generated sequence of the group assignment and numbers will be sealed in opaque envelopes and stored in double-locked cabinets. Allocation concealment will be maintained throughout the entire study.

\section{Blinding}

The present study will use a double-blind method. The placebos and JTW will look the same and will be packaged in uniform plastic bags. Then, the study coordinator will assign JTW or placebo according to the randomization codes and place them in special containers. The participants, outcome assessors, study coordinators, data managers, and statisticians will be blinded to the group allocation and numbered drug containers throughout the entire study. At the end of the study, the blinding codes will be revealed.

If unblinding is required, the trial managers and data coordinators will have access to group allocations and any unblinding will be reported.

\section{Intervention}

These eligible participants will ingest $2 \mathrm{~g}$ granules of JTW or placebo, twice a day at 1600 hours and 2100 hours for 1 week and present themselves at the termination of medication, followed by a scheduled examination.

Both JTW and placebo are manufactured by the Kangren Pharmaceutical Factory according to good manufacturing practice standards. JTW will be produced in the form of granules with a yellowish-brown color. The medication will be $2 \mathrm{~g}$, containing $1.1 \mathrm{~g}$ JTW soft extract with $0.37 \mathrm{~g}$ of lactose hydrate and $0.88 \mathrm{~g}$ of corn starch as the excipient. The JTW soft extract will be made of a water extract of a mixture of two herbal medicines, as follows: Rhizome Coptidis ( $10 \mathrm{~g})$ and Cortex Cinnamomi $(1 \mathrm{~g})$. The placebo will consist of corn starch, lactose hydrate, citric acid, and caramel color. The placebo and JTW will have the same appearance and color.

\section{Medication compliance monitoring}

To ensure compliance with the medication, participants will be asked to count the actual intake and return the empty wrapping papers for the medication and any remaining medicine. 


\section{Prohibition and permission for concomitant treatment}

1. The use of any sedative hypnotics to help sleep will be prohibited during the study.

2. The use of any traditional medicine designed to treat insomnia, except for the intervention of this trial, will be prohibited during the study.

3. The use of functional foods or other medications intended to improve the symptoms of insomnia will be prohibited during the study.

4. Any psychotherapy for accelerating sleep quality will be prohibited during the study.

5. Medications used to treat other chronic diseases at the beginning of this trial will be allowed.

6. Routine physical training before the start of this clinical trial will be allowed.

7. Any change in medications or physical exercise during the study will be recorded in a clinical report form.

\section{Outcome measurement \\ Primary outcome}

We chose 1 week to assess the curative effect of JTW based on: (1) our previous experimental results and findings on animal sedation and hypnosis [16]; and (2) our clinical experience with patients using JTW.

Pittsburgh Sleep Quality Index The PSQI will be used at the baseline and the end of the drug intervention. The PSQI version used in this study is a 19-item selfreported retrospective questionnaire to access the quality of sleep in the past 7 days, and it is designed to measure seven domains called component scores: subjective sleep quality; sleep latency; sleep duration; habitual sleep efficiency; sleep disturbances; use of sleep medications; and daytime dysfunction [16, 17]. Component scores range from 0 (no difficulty) to 3 points (severe difficulty), and when summed, produce a global score in the range of $0-21$. A higher score denotes worse sleep. These syndromes are categorized as mild (0-1), moderate (2-7), or severe $(\geq 8)$ [18]. Then, the scores the participants obtained at baseline will be recorded as score 0 , and the scores obtained at the end of drug intervention will be recorded as score 1 . The recorder will calculate the reduction rate (RR) according to the following formula: $\mathrm{RR}=($ score 1 -score 0$) /$ score $0 * 100 \%$. Finally, we will assess the clinical curative effect of every participant based on the results of the RR. RR values $>75 \%$ will be regarded as a clinical cure, RR values between $50 \%-75 \%$ will be considered as a clinical effective, RR values between $25 \%-50 \%$ will be recognized as a clinical success, and $R R$ values $<25 \%$ will be considered ineffective. The primary outcome is defined to have a value of 0 if $R R$ is $<25 \%$ (ineffective) and 1 if RR is $>25 \%$ (clinical cure, clinically effective, or clinical success). The proportion of 1 s (i.e. $R R \geq 25 \%$ ) will be compared between the treatment and placebo groups.

In addition, Liu et al. [18] presented their opinion that the PSQI is suitable for Chinese patients after they conducted reliability and validity tests [19].

\section{Secondary outcomes}

Polysomnography Polysomnography (PSG) will be taken twice, at both the baseline and the end of the drug intervention. On the first night, participants will adapt themselves to the laboratory environment. On the second night, participants will be placed on PSG monitoring to record the multiple physiological sleep parameters. The parameters $[20,21]$ will include total sleep time, sleep efficiency, sleep latency, rapid eye movement (REM) stage latency, wake after sleep onset, and the time duration of the particular sleep stages (such as N1, N2, $\mathrm{N} 3$ ). The mean changes of all the parameters from the baseline to the end of the drug intervention will be used to measure the changes in sleep quality.

PSG is considered the gold standard for scoring sleep disorders. The scoring of sleep and arousals relies on visual inspection of continuous surface electroencephalography (EEG), electromyography (EMG), and electrooculography (EOG) during PSG and then divides human sleep into five stages: wakefulness (W); REM; non-rapid eye movement (NREM); and N1, N2, and N3 [22]. The American Academy of Sleep Medicine (AASM) defined the quantitative reference standard in PSG for adult insomnia as follows [23]: sleep latency $\geq 30 \mathrm{~min}$; total sleep time $<390 \mathrm{~min}$; number of wake after sleep onset (WASO) $\geq 2$ or time of $\mathrm{WASO} \geq 40 \mathrm{~min}$; time in N1/total sleep time $>60 \%$; time in N2 / total sleep time $>60 \%$ or time in N3/total sleep time $<10 \%$, or time in REM / total sleep time $<20 \%$. A higher or lower score corresponds to more severe symptoms.

${ }^{\mathbf{1}} \mathrm{H}$-magnetic resonance spectroscopy ${ }^{1} \mathrm{H}$-magnetic resonance spectroscopy $\left({ }^{1} \mathrm{H}\right.$-MRS $)$ will also be taken at the baseline and the end of the drug intervention. ${ }^{1} \mathrm{H}$-MRS, with its unique non-invasive advantages, is able to detect and quantify the important metabolites of living brain tissue, including $\mathrm{N}$-acetylaspartate (NAA), choline (Cho), creatine $(\mathrm{Cr})$, gamma-aminobutyric acid (GABA), and myo-intositol (mI) [24]. In the present study, single voxel hippocampus and thalamus metabolite ratios of GABA with $\mathrm{Cr}$ will be measured. The altered ratios of GABA with $\mathrm{Cr}$ between baseline and the end of the drug intervention will be the second outcome measure.

Studies have revealed that the $\mathrm{GABA} / \mathrm{Cr}$ ratio in the frontal lobe is significantly lower [25], and the average 
brain GABA levels are nearly $30 \%$ lower in patients with primary insomnia [26].

Disharmony of Heart and Kidney Scoring System The Disharmony of Heart and Kidney Scoring System will be used at the baseline and the end of drug intervention. It is a checklist covering one indispensable and seven accompanying items. These items are the symptoms and signs of the disharmony of heart and kidney pattern according to the theory of the pattern identification system in traditional East Asia medicine. The score of the indispensable item uses a 4-point scale $(0,3,6$, and 9) depending on the severity of the insomnia $(0=$ none, $9=$ very severe). The seven accompanying items including palpitations, dizziness, spermatorrhea or menstrual irregularity, and night sweat and use scale of $0-4$ points based on their frequency. A total score of $>9$ out of 30 points is thought to demonstrate disharmony of the heart and kidney [27, 28].

Although there is no accurate evidence to explain the validity and reliability of this questionnaire, no other methods are currently available to identify the disharmony of heart and kidney pattern.

Blood tests Blood tests consists of the levels of adenosine $(\mathrm{AD})$ and melatonin ( $\mathrm{N}$-acetyl-5- methoxytryptamine) in blood samples will be recorded at the baseline and the end of the drug intervention and will then be analyzed by the clinical lab.

Sleep homeostasis in adults is affected by the sleepregulatory substances $\mathrm{AD}$ and melatonin $[29,30]$. $\mathrm{AD}$ is a product of brain metabolism and is closely related to sleep parameters. A previous study [31] showed that AD levels were elevated as a consequence of sustained wakefulness. Melatonin is an endogenous hormone produced by the pineal gland and is released exclusively at night. Melatonin has been shown to synchronize the circadian rhythms, and improve the onset, duration, and quality of sleep. Takaesu et al. thought that reduced secretion of melatonin may be involved in the mechanism of insomnia [32].

In the present study, high-performance liquid chromatography (HPLC) will be developed to determine the levels of adenosine, and an enzyme-linked immunosorbent assay will be used to detect the concentration of melatonin.

\section{Safety outcomes}

At the end of this trial, participants will participate in a routine physical examination consists of breath rate, heart rate, temperature, blood pressure, weight, routine urine, routine blood test, liver, kidney function tests, and electrocardiogram.

\section{Reporting and treating of adverse events}

During the treatment period, the researchers will record any adverse events (AEs) that will be defined as unpredictable, undesirable symptoms, signs, or diseases related to the treatment during daily telephone calls. The researchers will then comprehensively evaluate the correlation between these AEs and the experimental drugs according to the recorded details about AEs, including the manifestation, occurrence time, duration, cause, and treatment measures. Simultaneously, if any AE occurs, the investigator will take proper measures such as dose adjustment, drug withdrawal, and symptomatic treatment to ensure the safety of participants; all details will be written down carefully. Furthermore, continuous follow-up will be insisted upon until the condition of the participants returns to normal. Furthermore, an insurance contract will be concluded to cover financial compensation to participants who might be injured during the study.

\section{Participant drop-outs}

1. The participant can decide to discontinue treatment at any time for any reason.

2. The patient is non-compliant.

3. The patients experiences a serious AE.

Drop-outs and withdrawals from the trial will be recorded throughout the intervention and follow-up periods. Their data will be collected in the case report form but will not be used for the final statistical analysis.

\section{Sample size}

The ratio of cases between the JTW group and the placebo group is set to 1:1; this study is designed to achieve a statistical power of $80 \%$ (two-sided type- 1 error of $\alpha=$ $5 \%, \beta=20 \%$ ). Based on previous clinical practice [33, 34 , we assume a treatment success rate of $40 \%$ in the placebo group and $70 \%$ in the JTW group over 7 days. The sample size is calculated using the following formula:

$$
\begin{aligned}
N=[ & {\left[\Phi^{-1}(\alpha)+\Phi^{-1}(\beta / 2)\right]^{2} \times\left(\pi_{e}+\pi_{0}\right) } \\
& \times\left(100-\left(\pi_{e}+\pi_{0}\right) / 2\right) /\left(\pi_{e}-\pi_{0}\right)^{2}
\end{aligned}
$$

where $\Phi$ is the cumulative distribution function of the standard normal distribution, and $\pi_{e}$ and $\pi_{0}$ are the success rate in the experimental and control groups, respectively. Considering a $20 \%$ drop-out rate, a sample size of $N=49 /(1-0.2)=62$ is needed for each group.

Thus, under the assumptions of a success rate of $70 \%$ for treatment and $40 \%$ for placebo, and a noncompliance rate of $20 \%$, the sample size required to have at least $80 \%$ power of detecting a difference between the 
two groups at a two-sided alpha level of $5 \%$ is 124 participants.

\section{Data management and monitoring}

The experimental data will be carefully saved in Microsoft Access, a database management system (DBMS) from Microsoft. To guarantee the data quality, data will be input and checked twice by two researchers. During the study, the First Affiliated Hospital of Wenzhou Medical University is responsible for making regular visits (once a month) to review trial conduct; the Ethics Committee will monitor for protocol violations monthly. There was no conflict of interest with the sponsors or researchers.

In addition, confidentiality of participants' data is ensured by using participants' IDs rather than identifiable information in the dataset (i.e. coding) and by storing the document linking the IDs to the identifiable information separately and securely. Only researchers directly involved in the analysis of the randomized controlled trial (RCT) will have access to the final trial dataset, which will only contain coded data. After data collection and before data storage, all outcomes are manually double-checked by the research staff. The safety, progress, study integrity, and design aspects will be monitored at various meetings by the research team involved in this study.

\section{Statistical analysis}

We will use two-sided $p$ values for the primary outcome and for all secondary outcomes and safety variables. For the primary outcome, we will use Fisher's exact test to test the hypothesis at null: the treatment has the same efficacy as the placebo. For each secondary outcome, defined as the value difference between baseline and endof-intervention, we will use the following tests: (1) t-test for the continuous PSG variables; Chi-square test for the categorical PSG variables; (2) t-test for the blood-level adenosine and melatonin; (3) t-test for the GABA/Cr ratio; and (4) Wilcoxon rank sum test for the Disharmony Score of Heart and Kidney. For safety evaluation of JTW, a two-sided t-test will be applied to each safety measurement after data normalization.

We define the analysis population as the enrolled participants who have successfully completed the trial. We will characterize the types of individuals who have missing data to better understand the nature the missing mechanisms. We will use statistical models to impute missing values if the assumption of missing at random is met. This can preserve and utilize the information of the non-missing values.

\section{Discussion}

JTW, first appearing in an old classical text of ancient Chinese medicine [35], is a well-known tranquillizing formula to treat insomnia due to disharmony of the heart and kidney [36]. As mentioned, modern scientific studies have shown that JTW may be helpful for people suffering from insomnia [10-13]; however, to the best of our knowledge, this is the first RCT that will determine the efficacy and safety of JTW in the therapy of insomnia symptoms, which is why we intend to perform this study.

However, the present study has some limits. First, the study period is short, with short time follow-up times. Second, the pattern of insomnia in this study is relatively simple, with only the pattern of disharmony of the heart and kidney included. Lastly, a single-center sample study is less convincing than a multi-center sample study. Despite these limits, the present study is reasonably well designed. The results of the present study are expected to guide physicians or traditional medical doctors at clinics more clearly in prescribing JTW and to widely spread the use of traditional medicine in other countries.

\section{Trial status}

Protocol version date and number: February 10, 2020; version 1.3.

Patient recruitment began in September 2018 and it will be completed by December 2020 .

\section{Supplementary information}

Supplementary information accompanies this paper at https://doi.org/10 1186/s13063-020-04299-x.

Additional file 1. SPIRIT checklist.

\section{Abbreviations}

AASM: American Academy of Sleep Medicine; AD: Adenosine; AE: Adverse event; CCMD-3: Chinese Classification and Diagnostic Criteria of Mental Disorder-3 Version; Cho: Choline; Cr: Creatinine; EEG: Electroencephalography; EMG: Electromyography; EOG: Electrooculography; GABA: Gammaaminobutyric acid; ' ${ }^{1} \mathrm{H}$-MRS: ${ }^{1} \mathrm{H}$-magnetic resonance spectroscopy; HPLC: High performance liquid chromatography; JTW: Jiao-tai-wan; ml: Myo-inositol; NAA: N-acetylaspartate; NREM: Non-rapid eye movement; PSG: Polysomnography; PSQI: Pittsburgh Sleep Quality Index; RCT: Randomized controlled trial; REM: Rapid eye movement; RR: Reducing rate; SPIRIT: Standard Protocol Items: Recommendations for Interventional Trials; W: Wakefulness; WASO: Wake after sleep onset

\section{Acknowledgements}

Not applicable.

\section{Results dissemination}

The trial results will be communicated to participants, healthcare professionals, the public, and other relevant groups via Chinese Clinical Trial Registry (http://www.chictr.org.cn/).

\section{Authors' contributions}

YZZ and YR are the principal investigators and corresponding authors. ZCC and $L X$ are the first authors responsible for preparing the manuscript. ZCC is the research investigator. $L X$ is the second investigator. $H L F, X N Z$, and $Z H H$ are the outcome assessors. YZZ and YR conceived this trial and participated in its design. $\mathrm{LL}$ is responsible for data management and analysis. FY, ZCC, and LX will coordinate the trial and contribute to the screening of patients and are also involved in the recruitment of participants from clinics. ZCC will collate the data and prepared the manuscript. LX provided critical revision of the manuscript. All named authors adhere to the authorship guidelines of 
Trials. All authors have agreed to publication. Conceptualization: YZZ and YR. Formal analysis: a statistician from the Clinical Evaluation and Analysis Centre of the First Affiliated Hospital of Wenzhou Medical University. Investigation: ZCC, LX, and FY. Methodology: LL, HLF, XNZ, and ZHH. Supervision: YZZ and YR. Writing original draft: ZCC. Writing review and editing: LX.

\section{Funding}

This study is supported by the National Natural Science Foundation of China (no. 81673733). The funder had no role in the design of the study; analysis, collection, and interpretation of the data; or the writing and decision for publication of the manuscript.

\section{Availability of data and materials}

The datasets used or analyzed during the current study will be available from the corresponding author upon reasonable request.

\section{Ethics approval and consent to participate}

The study has been approved by the Ethical Research Committees of the First Affiliated Hospital of Wenzhou Medical University (2016045). Written informed consent will be obtained from all participants by the investigator. On the consent form, participants will be asked if they agree to use of their data should they choose to withdraw from the trial. Participants will also be asked for permission for the research team to share relevant data with people from regulatory authorities.

\section{Consent for publication}

Not applicable.

\section{Competing interests}

The authors declare that they have no competing interests.

\section{Author details}

'Department of Traditional Chinese Medicine, The First Affiliated Hospital of Wenzhou Medical University, Nanbaixiang, Ouhai District, Wenzhou 325035, Zhejiang, China. ${ }^{2}$ Zhejiang Chinese Medical University, Binwen Road, Binjiang District, Zhejiang 310053, Hangzhou, China. ${ }^{3}$ Department of Pharm, The First Affiliated Hospital of Wenzhou Medical University, Wenzhou 325035, Zhejiang, China. ${ }^{4}$ The 2 nd Clinical College of Wenzhou Medical University, Chashan Higher Education Park, Wenzhou 325035, Zhejiang, China. ${ }^{5}$ X-ray Department, The First Affiliated Hospital of Wenzhou Medical University, Wenzhou 325035, Zhejiang, China. ${ }^{\circ}$ Sleep monitoring center, The First Affiliated Hospital of Wenzhou Medical University, Wenzhou 325035, Zhejiang, China. ${ }^{7}$ Research Institute of China Academy of Chinese Medical Sciences, No.16 Nanxiao Street, Dongzhimen, Dongcheng District, Beijing 100700, China.

Received: 23 March 2019 Accepted: 1 April 2020

Published online: 15 May 2020

\section{References}

1. Ohayon M. Epidemiology participantsof insomnia: what we know and what we still need to learn. Sleep Med Rev. 2002;6:97-111.

2. Sadeghniiat-Haghighi K, Montazeri A, Khajeh-Mehrizi A, Nedjat S, Aminian O. The insomnia severity index: cross-cultural adaptation and psychometric evaluation of a Persian version. Qual Life Res. 2014;23:533-7.

3. Hertenstein E, Feige B, Gmeiner T, Kienzler C, Spiegelhalder K, Johann A, et al. Insomnia as a predictor of mental disorders: A systematic review and meta-analysis. Sleep Med Rev. 2019;43:96-105.

4. Morgenthaler T, Kramer M, Alessi C, Friedman L, Boehlecke B, Brown T, et al. Practice parameters for the psychological and behavioral treatment of insomnia: An update. An American academy of sleep medicine report. Sleep. 2006;29:1415-9.

5. Shi HI, Lin CC, Tu YF, Chang CM, Hsu HC, Chi CH, Kao CH. An increased risk of reversible dementia may occur after zolpidem derivative use in the elderly population: a population-based case-control study. Medicine (Baltimore). 2015;94(17):809-16.

6. Leach MJ, Page AT. Herbal medicine for insomnia: a systematic review and meta-analysis. Sleep Med Rev. 2015;24:1-12.

7. Poon MM, Chung KF, Yeung WF, Yau VH, Zhang SP. Classification of insomnia using the traditional Chinese medicine system: a systematic review. Evid Based Med Complement Alternat Med. 2012;2012:735078.
8. Yuan ZZ, Ye R, Xiang ZC, Na Z, He JC. The distribution pattern of TCM syndromes of insomnia in 913 patients. Chinese Journal of Traditional Chinese Medicine. 2011;29(7):1508-10.

9. Quan SJ, He SM, Qian LL. Study on mechanism of Jiao Tai Wan on treatment of insomnia by communication between heart and kidney. J Liaoning Univ TCM. 2011;13(8):12-4.

10. Yeung W, Chung K, Man-Ki Poon M, Yan-Yee Ho F, Zhang S, Zhang Z, TatChi Ziea E, Wong VT. Chinese herbal medicine for insomnia: a systematic review of randomized controlled trials. Sleep Med Rev. 2012;16(6):497-507.

11. Yang YT. The Randomized Parallel Controlled Study of Jiaotai Pill Acupoint Sticking Application Therapy for the Insomnia Patients which Diagnosed with the TCM Syndrome. J Pract Tradit Chin Intern Med. 2016;7:17-9.

12. Lu WZ, Xu DL, Wang QC. Clinical study of jiaotai pill in the treatment of diabetes mellitus with insomnia. J HEIBEI TCM Pharmacol. 2005;2:35-7.

13. Luo SW. The curative effect of Jiaotaiwan on insomnia. J Pract Tradit Chin Med. 2018;34:1162.

14. Chan AW, Tetzlaff JM, Altman DG, Laupacis A, Gotzsche PC, Krleza-Jeric K, et al. SPIRIT 2013 Statement: Defining Standard Protocol Items for Clinical Trials. Ann Intern Med. 2013;158(3):200-7.

15. Psychosis Branch of Chinese Medical Association. The Chinese Classification and the Diagnose Criterion of Mental Disorder (CCMD). 3rd ed. Jinan: Shandong Technology Press; 2001.

16. Ni XF, Lin SS, Chen Q, et al. Sedative effect on mice of Jiao-Tai-Wan with different concentrations and its compositions. J Wenzhou Med Univ. 2019; 49(9):639-43.

17. Buysse DJ, Reynolds CF, Monk TH, Berman SR, Kupfer DJ. The Pittsburgh Sleep Quality Index (PSQI): a new instrument for psychiatric research and practice. Psychiatrist Res. 1989;28:193-213.

18. Liu XC, Tang MQ, Hu L, et al. Reliability and validity of the Pittsburgh Sleep Quality Index. Chin J Psychiatry. 1996;29(2):103-7.

19. Smith MT, Wegener ST. Measures of sleep: the insomnia severity index, medical outcomes study (MOS) sleep scale, Pittsburgh sleep diary (PSD), and Pittsburgh Sleep Quality Index (PSQI). Arthritis Rheum. 2003;49:S184-9.

20. Manni R, Terzaghi M, Repetto A. The FLEP scale in diagnosing nocturnal frontal lobe epilepsy, NREM and REM parasomnias: data from a tertiary sleep and epilepsy unit. Epilepsia. 2008:49:1581-5.

21. Kushida CA, Littner MR, Morgenthaler T, Alessi CA, Bailey D, Coleman J Jr, et al. Practice parameters for the indications for polysomnography and related procedures: an update for 2005. Sleep. 2005;28:499-521.

22. Epstein LJ, Kristo D, Strollo PJ Jr, Friedman N, Malhotra A, Patil SP, et al. Clinical guideline for the evaluation, management and long-term care of obstructive sleep apnea in adults. J Clin Sleep Med. 2009;5(3):263-76.

23. American Academy of Sleep Medicine. International classification of sleep disorders. 3rd ed. Darien: American Academy of Sleep Medicine; 2014.

24. Jansen JF, Backes WH, Nicolay K, Kooi ME. 1H MR spectroscopy of the brain: absolute quantification of metabolites. Radiology. 2006;240:318-32.

25. Kakeda S, Korogi Y, Moriya J, Ohnari N, Sato T, Ueno S, Yanagihara N, Harada M, Matsuda T. Influence of work shift on glutamic acid and gammaaminobutyric acid (GABA): evaluation with proton magnetic resonance spectroscopy at 3T. Psychiatry Res. 2011;192(1):55-9.

26. Winkelman JW, Buxton OM, Jensen JE, Benson KL, O'Connor SP, Wang W, Renshaw PF. Reduced brain GABA in primary insomnia: preliminary data from 4T proton magnetic resonance spectroscopy ('H-MRS). Sleep. 2008; 31(11):1499-506.

27. Zheng XY. Clinical guideline of new drugs for traditional Chinese medicine. Beijing: Medicine Science and Technology Press of China; 1993.

28. State Administration of Traditional Chinese Medicine. Standards for diagnosis and curative effect of Chinese medical symptom. Nanjing: Nanjing University Publishing House; 1994.

29. Huang $Z \mathrm{~L}$, Urade $\mathrm{Y}$, Hayaishi $\mathrm{O}$. The role of adenosine in the regulation of sleep. Curr Top Med Chem. 2011;11(8):1047-57.

30. Singh M, Jadhav HR. Melatonin: functions and ligands. Drug Discov Today. 2014;19(9):1410-8.

31. Porkka-Heiskanen T, Strecker RE, Thakkar M, Bjorkum AA, Greene RW, McCarley RW. Adenosine: A Mediator of the Sleep-Inducing Effects of Prolonged Wakefulness. Science. 1997;276(5316):1265.

32. Takaesu Y, Futenma K, Kobayashi M, et al. A preliminary study on the relationships between diurnal melatonin secretion profile and sleep variables in patients emergently admitted to the coronary care unit. Chronobiol Int. 2015;32:875-9. 
33. Wang $\mathrm{H}$, Quan $\mathrm{S}$, Sheng Y. Effect of Jiaotai pill on serum cytokine in rats with insomnia induced by para-chloroamphetamine acid. Guangzhou Zhong Yi Yao Da Xue Xue Bao. 2008;25(6):525-710.

34. Dong XW. Clinical observation of 40 cases of insomnia treated with jiao-taiwan. Public Med Forum Mag. 2015;13(26):3680-1.

35. Committee of the Pharmacopoeia of China. Pharmacopoeia of PR China, Part I. Beijing; Chemical Industry Press; 2005.

36. Zhang H, Guo XZ. On Selection of Sedative Herbal Medicine from Theory of 'Disharmony between Heart and Kidney. World Chin Med. 2015;6(5):381-4.

\section{Publisher's Note}

Springer Nature remains neutral with regard to jurisdictional claims in published maps and institutional affiliations.

Ready to submit your research? Choose BMC and benefit from:

- fast, convenient online submission

- thorough peer review by experienced researchers in your field

- rapid publication on acceptance

- support for research data, including large and complex data types

- gold Open Access which fosters wider collaboration and increased citations

- maximum visibility for your research: over $100 \mathrm{M}$ website views per year

At $\mathrm{BMC}$, research is always in progress. 